Women's health

\section{Occupational exposure to glycol ethers and ovarian function}

\section{S Cordier, L Multigner}

\section{Commentary on the paper by Hsieh et al (see page 510)}

T simultaneously hydrophilic and lipophilic properties of glycol ethers account for their use as solvents in a wide range of industrial and domestic products, including paints, varnishes, cleaning agents, and cosmetics. These dozens of compounds are grouped into two main classes: ethylene glycol ethers (EGEs) and propylene glycol ethers (PGEs). Because animal experiments show several EGEs to have high potential for toxicity, especially for testicular function, there has been a shift since 1990 in European countries towards replacing short chain EGEs with PGEs. In various sectors, however, including the semiconductor industry, several short chain EGEs are still in use. ${ }^{1}$

Short chain EGEs, more specifically, ethylene glycol methyl ether (EGME), ethylene glycol ethyl ether (EGEE), and their acetates, were first shown to affect testicular function, and more precisely, spermatocytes in the pachytene stage, dose dependently in all species tested, resulting in reduced fertility. ${ }^{2}$ Several other EGEs have also shown testicular toxicity, although at higher doses. Epidemiological investigations have examined sperm quality and male fertility in groups of workers occupationally exposed to EGEs as shipyard painters, in metal casting processes, and in the semiconductor industry. Even though studies on sperm quality are generally limited by low participation rates, these findings indicate that glycol ether exposure appeared to affect sperm production. The wives of male production workers in the semiconductor industry, that is, those exposed to glycol ethers, show a slightly longer average time to pregnancy compared to non-production workers. ${ }^{3}$

The reproductive toxicity of these compounds among females has been the object of much less study. A few animal studies show ovarian cycle perturbations secondary to luteal cell alterations and diminished fertility potential. Methoxyacetic acid (MAA), EGME's principal metabolite, increases progesterone production in cultured human luteal cells at the same concen- tration that increases progesterone in rat luteal cells in vivo. Evidence in humans is even more sparse. In this issue, Hsieh and colleagues ${ }^{4}$ report on altered prolonged menstrual cycles in female employees in the semiconductor industry in Taiwan. This investigation is a complement to and a possible explanation of a previous association in the same population. ${ }^{5}$ Previously, two large US studies in the semiconductor industry (the Semi-Conductor Health Study $(\mathrm{SHS})^{6}$ and the IBM study $^{3}{ }^{7}$ ) used prospective follow up of conceptions and clinically recognised pregnancies among women exposed to glycol ethers to study menstrual functions and fecundability. Their results do not lend themselves to clear-cut interpretation and were unfortunately not substantiated by the simultaneous biological assessment of exposure to glycol ethers, which should have been useful in disentangling the many coexposures.

Male reproductive insults have been studied in a variety of occupational settings, but most studies of women have taken place in the semiconductor industry, which is also characterised by exposure to mixtures of other compounds (arsenic, isopropyl alcohol, hydrofluoric acid, phosphorous compounds, xylene, etc), several suspected of reproductive toxicity. Both co-exposures and extent of EGEs use differ between work areas, and in most studies in this industry, risk classification according to work area has been used as a proxy for assessment of EGEs exposure. Risks are suspected to be highest in the photolithography sector; this is one of the two areas where Hsieh and colleagues ${ }^{4}$ found the highest risk of long menstrual periods, together with the diffusion area, where EGEs are much less frequent. The authors suggest that exposure to isopropyl alcohol may also play a role in the increased risks they found. The US studies also found contradictory results for risks according to work area. Classification according to semiquantitative levels of glycol ether exposure shows an association with with prolonged time to pregnancy found fecundability in the prospective study of conception in the SHS study but not the IBM study. Only rarely have urinary levels of EGEs metabolites been used to characterise exposure. In one small study, these metabolites showed no association with menstrual cycle duration. ${ }^{8}$

Studies of women's reproductive health in relation to environmental or occupational exposures have rarely looked at menstrual cycle characteristics or ovarian function, probably because of the specific difficulties associated with this evaluation. Measurement tools range from simple questionnaire reports of menstrual cycle characteristics to daily collection of urine samples to determine hormone levels. Systematic study of factors affecting menstrual cycle characteristics is only now beginning. ${ }^{9}$ For these reasons, it is hard to decide whether the contradictory results observed in different populations exposed to similar compounds are inherent to the outcome under study and the uncertainties in its measurement, or to random fluctuation. For instance, both short $(<24$ days in the SHS study $)^{6}$ and long $(>35$ days in Hsieh and colleagues' report) ${ }^{4}$ menstrual cycles have been observed in association with glycol ether exposure. Recent evaluations of the impact of other chemicals, such as organochlorine compounds, on menstrual function have also yielded contradictory results..$^{11}$ These discrepancies underline the need for additional information about validated tools to evaluate ovarian function properly, by measuring different endpoints including steroid hormone levels to determine follicular and luteal phases, and to analyse their validity and feasibility for use in epidemiological assessment.

Occup Environ Med 2005;62:507-508.

doi: 10.1136/oem.2005.019901

\section{Authors' affiliations}

S Cordier, L Multigner, Inserm U625,

University of Rennes, Rennes, France

Correspondence to: Dr S Cordier, Inserm U625, University of Rennes, Rennes, France; sylvaine.cordier@rennes.inserm.fr

Competing interests: none declared

\section{REFERENCES}

1 Koh D, Chan G, Yap E. World at work: the electronics industry. Occup Environ Med 2004;61:180-3

2 INSERM. Expertise Collective. Ethers de Glycol. Quels risques pour la santé? Paris: INSERM 1999

3 Correa A, Gray RH, Cohen R, et al. Ethylene glycol ethers and risks of spontaneous abortion and subfertility. Am J Epidemiol 1996;143:707-17 
4 Hsieh GY, Wang JD, Cheng TJ, et al. Prolonged menstrual cycles in female workers exposed to ethylene glycol ethers in the semiconductor manufacturing industry Occup Environ Med 2005:62:510-16.

5 Chen PC, Hsieh GY, Wang JD, et al. Prolonged time to pregnancy in female workers exposed to ethylene glycol ethers in semiconductor manufacturing. Epidemiology 2002:13:191-6.

6 Schenker MB. Reproductive and other health effects of semiconductor work: the
Semiconductor Health Study. Am J Ind Med 1995;28:635-7

7 Gray RH, Correa A, Hakim RR, et al. Ethylene glycol ethers and reproductive health in semiconductor workers. Occup Hyg 1996;2:331-8

8 Chia SE, Foo SC, Khoo NY, et al. Menstrual patterns of workers exposed to low levels of 2-ethoxyethylacetate (EGEEA). Am J Ind Med 1997;31:148-52.
9 Liu Y, Gold EB, Lasley BL, et al. Factors affecting menstrual cycle characteristics. Am J Epidemiol 2004:160:131-40.

10 Windham GC, Lee D, Mitchell $P$, et al. Exposure to organochlorine compounds and effects on ovarian function. Epidemiology 2005; 16:182-90.

11 Cooper GS, Klebanoff MA, Promislow J, et al Polychlorinated biphenyls and menstrual cycle characteristics. Epidemiology 2005; 16:191-200.

bias makes the use of self report problematic, however, unless there is special care taken to standardise infor-

\section{Self report and GIS based modelling as indicators of air pollution exposure: is there a gold standard?}

\section{F Forastiere, C Galassi}

\section{Commentary on the paper by Heinrich et al (see page 517)}

$\mathrm{E}$ xposure to traffic generated pollutants, especially among people living along busy roads, has been associated with increased risk of respiratory disorders among children and adults as well as with overall mortality. ${ }^{1-5}$ Simple exposure indicators (self report, distance from pollutant sources, traffic density) and more complex and integrated models that take into account demographic factors and land use by means of geographic information system (GIS) based technology $y^{6-8}$ have been employed in epidemiological studies.

As has happened in the past in occupational epidemiology, environmental epidemiologists are trying to develop more accurate methods for assessing air pollution exposure. ${ }^{9}$ As a result, comparing the different methods available is of great interest. In this issue, Heinrich et al have compared parental report of traffic intensity near homes (cars, trucks, buses, and mopeds on the street of residence and other nearby streets) with a combination of air pollution measures (fine particle mass, filter absorbance, and nitrogen dioxide) and GIS based modelling in two different European locations (the Netherlands and Munich, Germany). ${ }^{10}$ They found that predicted exposure estimates for air pollutants increased with self reported traffic level in Munich and in urban areas of the Netherlands. However, agreement rates between the two methods were relatively low for all three pollutants studied, and subjective assessments consistently overestimated fine particulate matter and nitrogen dioxide, in both locations and particularly in rural areas. The authors underline the limited validity of self reported traffic intensity measures.

From the results of this study should we conclude that subjective measures are bad and should not be used any more? Are the "objective" GIS based modelling methods the gold standard to recommend? Let us look at the main points.

The best way to learn about a specific fact, at least in theory, is to ask the most informed subject-that is, the person who is experiencing the exposure at issue, especially when his judgement can integrate several aspects like exposure intensity, distance from the source, and protective and remedial measures. Several well designed case-control studies in the occupational setting have used self reported exposures to toxic substances for this purpose. ${ }^{11}$ Beyond real exposure, however, self report is influenced by several other factors that may vary from one subject to another: individual perception, annoyance, socioeconomic and cultural background, and perhaps environmental "worry". The propensity to report more illnesses and symptoms as a result of proximity to a potential hazard, in the absence of a measurable biological effect, has been named "awareness bias". ${ }^{12}$ Moffat and colleagues $^{12}$ showed the influence of such awareness bias in two epidemiological studies conducted in communities exposed to emissions from heavy industry. The influence of the disease status on the report of exposure has not been described, to our knowledge, in air pollution studies. The possibility of this mation collection, to use simple and easy to understand terms, to employ relative or "objective" benchmarks, and to evaluate repeatability of the questionnaire.

The need to apply individual exposure estimates to air pollutants to large study populations has motivated the use of GIS in which geographic data are combined with concentration measurements to estimate exposures for subjects of large populations. ${ }^{67}$ In the original methodological study reported by Brauer and colleagues, ${ }^{8}$ and described here by Heinrich et al, 40 measurement sites within each of the two study areas provided annual average pollutant concentrations. Regression models predicting these concentrations were developed on the basis of population density and traffic intensity within buffer zones up to 1000 metres. The variability explained from the regression models using the 40 sites were $73 \%$ and $56 \%$ for $\mathrm{PM}_{2.5}$, respectively for the two locations, and $81 \%$ and $67 \%$, for filter absorbance (a marker of diesel exhaust). ${ }^{8}$ Air pollutant concentrations at the home address of the study subjects were estimated from these models. The performances of the models were good enough to render the method very attractive for exposure assessment. However, it is also evident that the "predicted exposure" for any individual subject in the study is affected by error and cannot be considered "the gold standard". Moreover, as the authors are well aware, GIS data cannot account for local scale differences when the grid size available is large because traffic related air pollutants exhibit substantial variability at distances of $50 \mathrm{~m}$ or less from major roads. Finally, exposures to some primary pollutants emitted from vehicular traffic may be overlooked with this method.

The past debate over exposure assessment in occupational epidemiology has some analogies. ${ }^{9}$ Work histories and job titles are of limited value in many instances and it may be difficult to obtain unbiased and valid estimation of occupational exposures in large 
studies relying on questionnaires. Alternative methods have been proposed, such as job exposure matrices (JEMs) and expert assessment. In particular, JEMs have been developed to study cancer, asthma, and, more recently, ergonomic problems. Although there is a large variability in validity and reliability estimates, it seems that self report tends to be a more sensitive method, whereas JEM seems to be more specific. Industrial hygienists, chemists, or engineers may be employed to estimate exposures of workers, with better performance than simple self report. However, there is a tendency towards integrating the methods available. In particular, self report can provide important information for expert judgement. With more complex and integrated exposure assessments a higher degree of reliability is obtained.

In conclusion, the results presented by Heinrich et al are very welcome, although they are not surprising, given the inherent limitations of the compared methods. Since no single method can be considered the "gold standard", it is clear that there is still room for further development in exposure assessment to air pollutants, possibly by integrating the information available from different sources, including the subjects under study. Such development will lead to more sophisticated approaches but perhaps, as in the occupational setting, ${ }^{13}$ to indications that simple indicators may be sufficient to obtain a valid study.

\section{ACKNOWLEDGEMENTS}

We thank Margaret Becker for her editorial help.

Occup Environ Med 2005;62:508-509. doi: 10.1136/oem.2005.020560

Authors' affiliations

F Forastiere, Department of Epidemiology, Rome E Health Authority, Rome, Italy C Galassi, Cancer Epidemiology Unit, CeRMS and Center for Cancer Prevention, University of Turin, Italy

Correspondence to: Dr F Forastiere, Department of Epidemiology, Rome E Health Authority, Via Santa Costanza, 53, 00198

Rome; forastiere@asplazio.it

Competing interests: none declared

\section{REFERENCES}

1 Ciccone G, Forastiere F, Agabiti N, et al. Road traffic and adverse respiratory effects in children. SIDRIA Collaborative Group. Occup Environ Med 1998;55:771-8.

2 Nicolai T, Carr D, Weiland SK, et al. Urban traffic and pollutant exposure related to respiratory outcomes and atopy in a large sample of children. Eur Respir J 2003;21:956-63.
3 Janssen NAH, Brunekreef $B$, van Vliet $P$, et al. The relationship between air pollution from heavy traffic and allergic sensitization, bronchial hyperresponsiveness, and respiratory symptoms in Dutch schoolchildren. Environ Health Perspect 2003;111:1512-18.

4 Garshick E, Laden F, Hart JE, et al. Residence near a major road and respiratory symptoms in U.S. Veterans. Epidemiology 2003; 14:728-36.

5 Hoek G, Brunekreef B, Goldbohm S, et al. Association between mortality and indicators of traffic-related air pollution in the Netherlands: a cohort study. Lancet 2002;360:1203-9.

6 Bellander T, Berglind N, Gustavsson P, et al. Using geographic information systems to assess individual historical exposure to air pollution from traffic and house heating in Stockholm. Environ Health Perspect 2001;109:633-9.

7 Briggs DJ, de Hoogh C, Gulliver J, et al. A regression-based method for mapping trafficrelated air pollution: application and testing in four contrasting urban environments. Sci Total Environ 2000;253:151-67.

8 Braver M, Hoek G, van Vliet P, et al. Estimating long-term average particulate air pollution concentrations: application of traffic indicators and geographic information systems. Epidemiology 2003;14:228-39.

9 Nieuwenhuijsen MJ. Exposure assessment in occupational and environmental epidemiology. London: Oxford University Press, 2003.

10 Heinrich J, Gehring U, Cyrys J, et al. Exposure to traffic related air pollutants: self reported traffic intensity versus GIS modelled exposure. Occup Environ Med 2005;62:517-23.

11 Blair A, Hayes RB, Stewart PA, et al. Occupational epidemiologic study design and application. Occup Med 1996;1 1:403-19.

12 Moffatt S, Mulloli TP, Bhopal R, et al. An exploration of awareness bias in two environmental epidemiology studies. Epidemiology 2000;11:199-208.

13 Axelson $\mathrm{O}$, Westberg $\mathrm{H}$. Introductory note on the concepts of exposure and dose in occupational epidemiology. Am J Ind Med 1992;21:3-4. 